\title{
FEASIBILITY OF MULTISPECTRAL AIRBORNE LASER SCANNING FOR LAND COVER CLASSIFICATION, ROAD MAPPING AND MAP UPDATING
}

\author{
Leena Matikainen*, Kirsi Karila, Juha Hyyppä, Eetu Puttonen, Paula Litkey, Eero Ahokas \\ Finnish Geospatial Research Institute FGI at the National Land Survey of Finland, Centre of Excellence in Laser Scanning \\ Research, Geodeetinrinne 2, FI-02430 Masala, Finland - (leena.matikainen, kirsi.karila, juha.hyyppa, eetu.puttonen, paula.litkey, \\ eero.ahokas)@nls.fi
}

Commission III, WG III/4

KEY WORDS: Laser scanning, lidar, multispectral, land cover, road, building, updating, change detection

\begin{abstract}
:
This article summarises our first results and experiences on the use of multispectral airborne laser scanner (ALS) data. Optech Titan multispectral ALS data over a large suburban area in Finland were acquired on three different dates in 2015-2016. We investigated the feasibility of the data from the first date for land cover classification and road mapping. Object-based analyses with segmentation and random forests classification were used. The potential of the data for change detection of buildings and roads was also demonstrated. The overall accuracy of land cover classification results with six classes was $96 \%$ compared with validation points. The data also showed high potential for road detection, road surface classification and change detection. The multispectral intensity information appeared to be very important for automated classifications. Compared to passive aerial images, the intensity images have interesting advantages, such as the lack of shadows. Currently, we focus on analyses and applications with the multitemporal multispectral data. Important questions include, for example, the potential and challenges of the multitemporal data for change detection.
\end{abstract}

\section{INTRODUCTION}

Multispectral airborne laser scanning (ALS) has recently become available and provides active multispectral information for 3D ALS point clouds from a single sensor. This technique is an interesting alternative to those commonly used in mapping, i.e., single-channel ALS and passive multispectral aerial imaging.

The first operational multispectral ALS system was launched by Teledyne Optech (Ontario, Canada) in late 2014 with the product name Titan. The sensor has three channels: infrared $1550 \mathrm{~nm}$ (Channel 1), near-infrared $1064 \mathrm{~nm}$ (Channel 2) and green $532 \mathrm{~nm}$ (Channel 3). Each channel produces a separate point cloud. The first studies based on Optech Titan data show the high potential of the data for applications such as land cover classification (e.g., Wichmann et al., 2015; Bakuła et al., 2016; Fernandez-Diaz et al., 2016; Matikainen et al., 2017; Morsy et al., 2017; Teo and Wu, 2017), road mapping (Karila et al., 2017), and map updating (Matikainen et al., 2017).

For our study, Optech Titan multispectral ALS data over a large suburban area in Espoo, Finland were acquired on three different dates: 21 August 2015 (leaves on), 2 May 2016 (leaves off), and 14 June 2016 (leaves on). Until now, our study has mainly focused on the feasibility of the data from the first date for land cover classification and road mapping. The potential of the data for change detection of buildings and roads was also demonstrated. The objective of the present article is to summarise our results and experiences with the multispectral data. The full details of the study have been published in Karila et al. (2017) and Matikainen et al. (2017).
Finally, we will briefly discuss our ongoing research with the multitemporal multispectral data.

\section{METHODOLOGY}

The multispectral Titan data were first preprocessed, which included, for example, radiometric correction and creation of digital surface models (DSMs), digital terrain models (DTMs) and three-channel intensity images in raster format. Objectbased analyses with segmentation and random forests (RF) classification were then used to investigate the feasibility of the data for land cover classification and road mapping. We selected object-based analysis of raster format data for this study because it is well suited for producing land cover classifications of large areas. Image format is also a natural way to present the intensity information and to compare it with passive aerial ortho images.

Segmentation was carried out by using the multiresolution segmentation algorithm (Baatz and Schäpe, 2000) in the eCognition software (Trimble Germany GmbH, Munich). Previous experience and visual analyses were used to select segmentation procedures and parameters. For the land cover classification tests, segmentation was first carried out based on a DSM. Low areas (mean heights less than $2.5 \mathrm{~m}$ from the ground surface) were then resegmented based on the threechannel intensity image. According to visual evaluation, the segmentation results were generally satisfactory. However, minor errors such as occurrence of some vegetation inside building segments were not completely avoided. eCognition

\footnotetext{
Corresponding author
} 
was also used in calculation of various features for the segments.

Feature importance analyses based on the RF method (Breiman, 2001), training data and various intensity and DSM features were then carried out. Finally, land cover and road classifications were performed, and the quality of the results was evaluated with independent validation data. Matlab software (The Mathworks, Inc., Natick, MA, USA) was used for the RF analyses and classification. The size of our study area was about $10 \mathrm{~km}^{2}$ in total. The reference data for training and validation were based on about 900 test field points from various land cover classes. Road detection and road surface classification were studied using extended training and validation point sets. The road detection and classification results were also compared to corresponding classifications based on aerial ortho imagery.

Automatic change detection for buildings and roads was first demonstrated by utilising the new multispectral ALS data in combination with old map vectors. In change detection of buildings, an old DSM based on single-channel ALS data was also used. Preliminary change detection tests with multitemporal multispectral data have also been carried out.

\section{RESULTS, VALIDATION AND DISCUSSION}

Figure 1 shows a Titan intensity image, a conventional aerial ortho image, and land cover classification results for a subarea of our study area. It can be seen that roads near buildings or trees are better visible on the Titan image than on the ortho image, although the Titan data are from leaf-on conditions and the ortho image is from leaf-off conditions. An important reason for this is the lack of shadows in the ALS data.

The land cover classification results are based on height and intensity information from the multispectral ALS data. The accuracy estimates for the land cover classification results are presented in Table 1 . These are based on 564 validation points from our entire study area. The accuracy estimates presented in Table 1 and other results from the object-based RF analysis suggested that the multispectral ALS data are very useful for land cover classification, considering both elevated classes and ground-level classes. Compared to classification of singlechannel ALS data, the main improvements were achieved for ground-level classes. According to feature importance analyses, multispectral intensity features based on several channels were more useful than those based on one channel.

In the additional road classification (see Figure 2), a total of 697 training points and 7346 validation points were used. Using multispectral ALS data $80.5 \%$ of the validation points were correctly classified as roads. Using aerial imagery the percentage was $71.6 \%$. Road surface was classified correctly into gravel or asphalt for $93.7 \%$ of the points when multispectral ALS was used. When aerial images were used, $84.8 \%$ of the points were classified correctly.
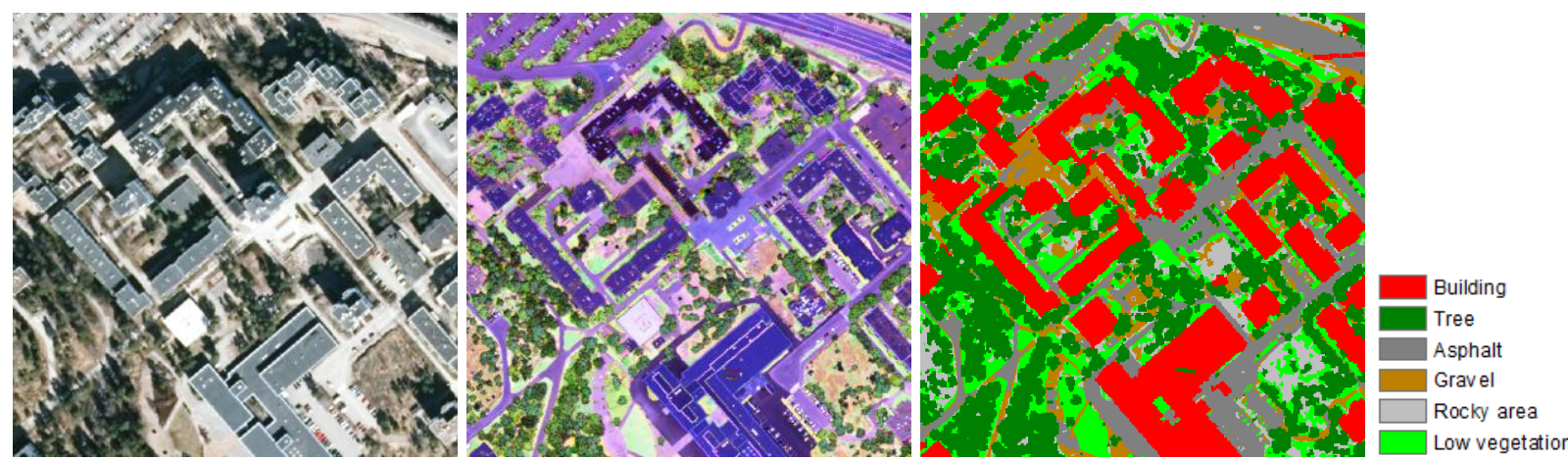

Figure 1. An aerial ortho image (left), Titan intensity image (middle), and land cover classification result (right) from a $300 \mathrm{~m} \times$ $300 \mathrm{~m}$ subarea. Ortho image (C) National Land Survey of Finland (NLS) 2013. The land cover classification is based on the multispectral ALS data.

\begin{tabular}{|l|c|c|}
\hline Class & Completeness & Correctness \\
\hline Building & $96.2 \%$ & $100 \%$ \\
\hline Tree & $100 \%$ & $97.9 \%$ \\
\hline Asphalt & $94.9 \%$ & $97.4 \%$ \\
\hline Gravel & $80.0 \%$ & $66.7 \%$ \\
\hline Rocky area & $90.9 \%$ & $90.9 \%$ \\
\hline Low vegetation & $96.1 \%$ & $92.4 \%$ \\
\hline Overall accuracy 95.9\% Kappa coefficient $\mathbf{0 . 9 5}$ \\
\hline
\end{tabular}

Table 1. Accuracy estimates for the land cover classification.

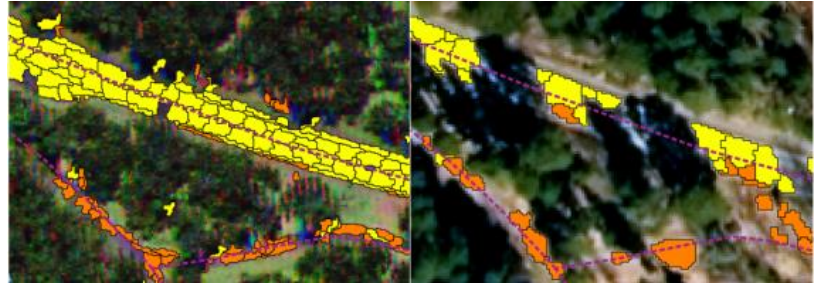

Figure 2. An example of road detection and classification results (left: multispectral ALS; right: aerial ortho image). Yellow: asphalt; orange: gravel. Dashed lines are road data from the NLS Topographic Database 2016. Aerial image () NLS 2013. 
Change detection of buildings and roads was demonstrated in a new residential area with plenty of changes (see Figure 3). The availability of two DSMs (the old one from single-channel ALS and the new one from multispectral ALS) allowed straightforward height comparisons for existing buildings to detect demolitions and buildings with changes in height. The multispectral information was exploited in the detection of new buildings. The method detected major changes such as new residential buildings well. For difficult cases such as checking small buildings in the forest, more sophisticated methods should be developed. In the case of roads, the special capability of the multispectral ALS data for ground surface classification was exploited. The change detection was simply based on the comparison of the asphalt and gravel surfaces from the land cover classification with old road vectors.

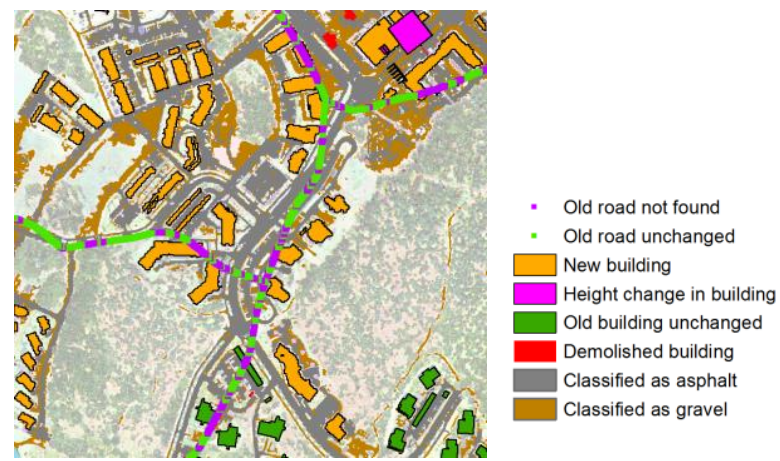

Figure 3. Change detection results for buildings and roads overlaid on the Titan intensity image in a new residential area. Original map data for old buildings (C) the City of Espoo. Original map data for old roads is based on the NLS Topographic Database 2000.

In our current work, we are analysing the potential of the multitemporal multispectral ALS data for direct height and intensity comparisons, which is likely to allow more straightforward change detection. Figure 4 shows a preliminary change detection result for an area where several trees have been cut due to the beginning of construction work. Change detection in this case was based on height comparison between DSMs from August 2015 and June 2016. A segment-based approach was used, and the classification result from August 2015 was also taken into account in the analysis.

When changes in intensity are considered, an important question is the stability of the intensity values between different scannings. Our first experiences show that there can be clear differences in the intensity values (Figure 5). Intensity of vegetated areas is expected to change during the year, but there are also changes in the intensity values of buildings and roads. For example, intensity values in August 2015 were higher than in the two other scannings, especially in Channel 1. Therefore, for change detection based on the intensity data, the intensity data should be calibrated or relative features (channel ratios) should be used. Further studies on the intensity changes are needed.

In addition to change detection, there are also other important research questions related to the multitemporal multispectral data. One such question is the effect of the data acquisition date on the usefulness of the intensity information. For example, data acquisition in leaf-off instead of leaf-on conditions is likely to have some advantages and some disadvantages in land cover classification.

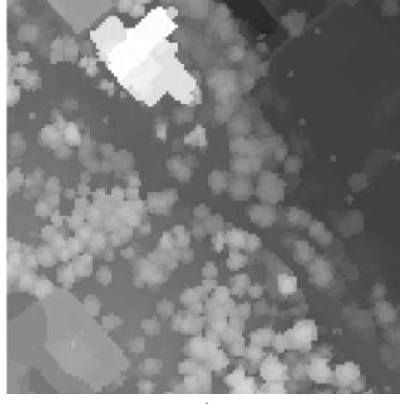

a)

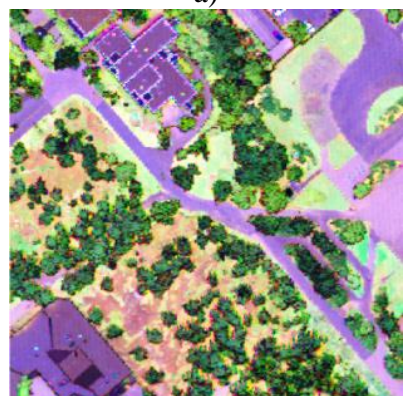

c)

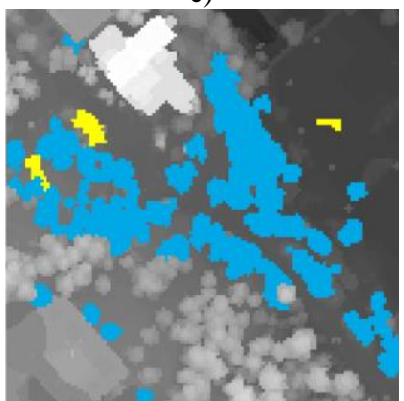

e)

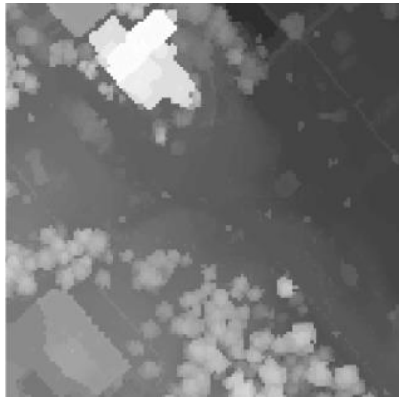

b)

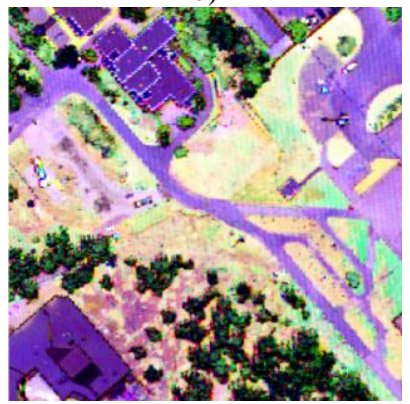

d)
Previously tree, height decrease $>2.5 \mathrm{~m}$ Previously ground, height increase $>2.5 \mathrm{~m}$

Figure 4. a) DSM from August 2015; b) DSM from June 2016; c) intensity image from August 2015; d) intensity image from June 2016; e) height changes detected in the area.

\section{CONCLUSIONS}

Our analyses suggest that the new multispectral ALS data have high potential for further increasing the automation level in mapping. Unlike passive aerial imaging, the technology is independent of external illumination conditions, and there are no shadows on intensity images produced from the data. The overall accuracy of land cover classification results with six classes was $96 \%$ compared with validation points. The data also showed high potential for road detection, road surface classification and change detection. In road detection and classification, the results were clearly better than corresponding results based on aerial ortho imagery. The availability of multitemporal multispectral data offers interesting possibilities for further development. 


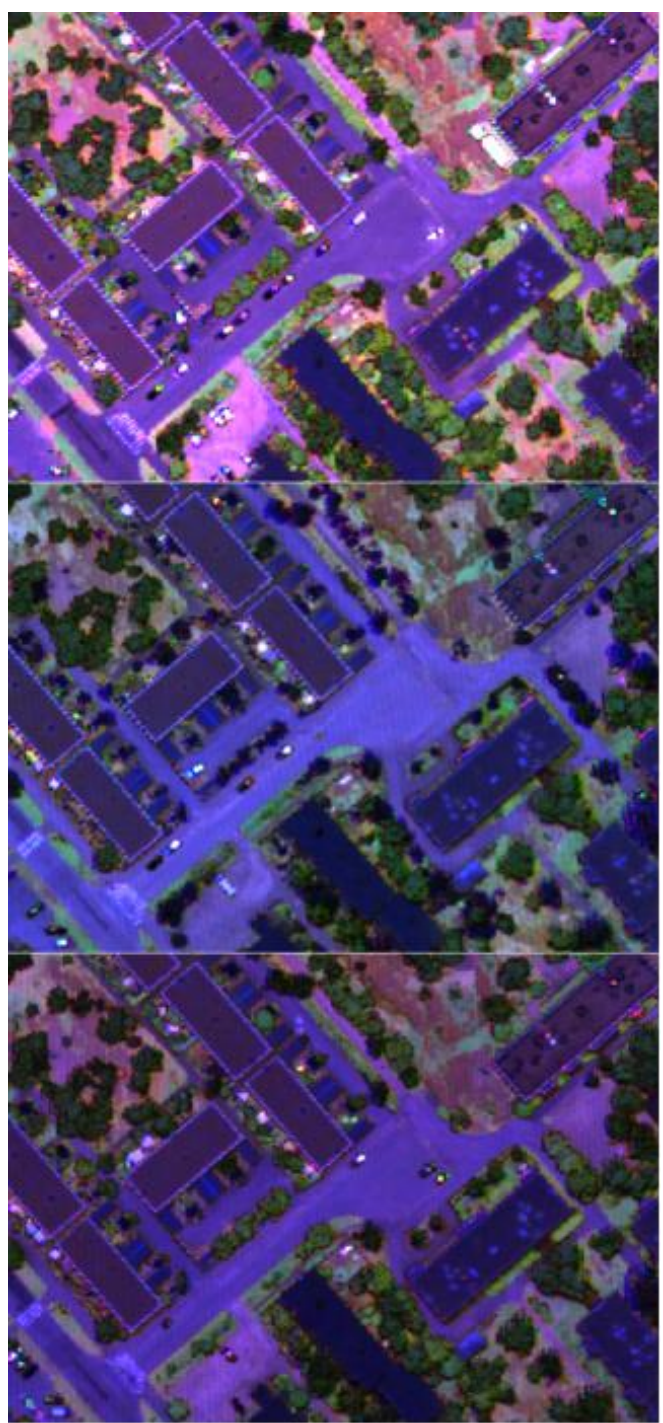

Figure 5. First pulse intensity images (20 cm raster) from August 2015 (top), May 2016 (middle) and June 2016 (bottom). In all images, the histogram stretching is the same.
Bakuła, K., Kupidura, P., Jełowicki, Ł., 2016. Testing of land cover classification from multispectral airborne laser scanning data. In: International Archives of Photogrammetry, Remote Sensing and Spatial Information Sciences, Prague, Czech Republic, Vol. XLI-B7, pp. 161-169.

Breiman, L., 2001. Random forests. Machine learning, 45(1), pp. 5-32.

Fernandez-Diaz, J.C., Carter, W.E., Glennie, C., Shrestha, R.L., Pan, Z., Ekhtari, N., Singhania, A., Hauser, D., Sartori, M., 2016. Capability assessment and performance metrics for the Titan multispectral mapping lidar. Remote Sensing, 8(11), 936.

Karila, K., Matikainen, L., Puttonen, E., Hyyppä, J., 2017. Feasibility of multispectral airborne laser scanning data for road mapping. IEEE Geoscience and Remote Sensing Letters, 14(3), pp. 294-298.

Matikainen, L., Karila, K., Hyyppä, J., Litkey, P., Puttonen, E., Ahokas, E., 2017. Object-based analysis of multispectral airborne laser scanner data for land cover classification and map updating. ISPRS Journal of Photogrammetry and Remote Sensing, 128, pp. 298-313.

Morsy, S., Shaker, A., El-Rabbany, A., 2017. Multispectral LiDAR data for land cover classification of urban areas. Sensors, 17, 958.

Teo, T.-A., Wu, H.-M., 2017. Analysis of land cover classification using multi-wavelength LiDAR system. Applied Sciences, 7, 663.

Wichmann, V., Bremer, M., Lindenberger, J., Rutzinger, M., Georges, C., Petrini-Monteferri, F., 2015. Evaluating the potential of multispectral airborne lidar for topographic mapping and land cover classification. In: ISPRS Annals of the Photogrammetry, Remote Sensing and Spatial Information Sciences, La Grande Motte, France, Vol. II-3/W5, pp. 113-119.

\section{ACKNOWLEDGEMENTS}

The authors would like to thank TerraTec Oy for cooperation in Optech Titan data acquisition, the NLS Department of Production for aerial ortho images and map data, and the City of Espoo for map data. The financial support from the Academy of Finland (projects 'Integration of Large Multisource Point Cloud and Image Datasets for Adaptive Map Updating' (project decision number 295047) and 'Centre of Excellence in Laser Scanning Research' (272195)) is gratefully acknowledged.

\section{REFERENCES}

Baatz, M., Schäpe, A., 2000. Multiresolution segmentation an optimization approach for high quality multi-scale image segmentation. In: Strobl, J., Blaschke, T., Griesebner, G. (Eds.), Angewandte Geographische Informationsverarbeitung XII: Beiträge zum AGIT-Symposium Salzburg 2000. Wichmann, Heidelberg, pp. 12-23. 